\title{
Preoperative short physical performance battery as predictors of prolonged hospitalization after coronary artery bypass grafting in older patients: a prospective cohort study
}

\author{
Peipei Han \\ Shanghai University of Medicine and Health Sciences \\ Liyuan Fu \\ Tianjin Medical University \\ XiaoYu Chen \\ Tianjin Medical University \\ Hairui Yu \\ Tianjin Medical University \\ Lin Hou \\ Tianjin Medical University \\ Lu Wang \\ Tianjin Medical University \\ Xing Yu \\ Tianjin Medical University \\ Jing Li \\ TEDA International Cardiovascular Hospital \\ Xiangjing Liu \\ TEDA International Cardiovascular Hospital \\ Bohan Shao \\ TEDA International Cardiovascular Hospital \\ Bo Yuan \\ TEDA International Cardiovascular Hospital \\ Min Li \\ TEDA International Cardiovascular Hospital \\ Hong Cheng \\ TEDA International Cardiovascular Hospital \\ Lei Teng \\ TEDA International Cardiovascular Hospital \\ Mingdi Guo \\ TEDA International Cardiovascular Hospital \\ Zhengqing Wang \\ TEDA International Cardiovascular Hospital \\ Tienan Chen \\ TEDA International Cardiovascular Hospital \\ Jianjun Liu \\ TEDA International Cardiovascular Hospital \\ Yaping Liu \\ TEDA International Cardiovascular Hospital \\ Zhigang Liu
}


TEDA International Cardiovascular Hospital

Xiaocheng Liu

TEDA International Cardiovascular Hospital

Qi Guo ( $\nabla$ guoqijp@gmail.com )

Shanghai University of Medicine and Health Sciences https://orcid.org/0000-0002-0193-4388

\section{Research article}

Keywords: 4-meter gait speed, coronary artery bypass grafting, elderly patients, prolonged hospitalization, short physical performance battery

Posted Date: December 10th, 2019

DOI: https://doi.org/10.21203/rs.2.18403/v1

License: (c) (i) This work is licensed under a Creative Commons Attribution 4.0 International License. Read Full License 


\section{Abstract}

\section{Background}

The short physical performance battery (SPPB) has played an important role in establishing a linkage between body function and health. Aims The aim of this study was to evaluate the predictive value of the SPPB summary score and its three components for screening prolonged hospitalization for coronary artery bypass grafting (CABG) in older patient candidates.

Methods

This study included 188 consecutive elderly patients (aged $\geq 60$ years) undergoing elective CABG surgery and all the patients were assessed for SPPB. Stepwise regression was employed to analyze the association of postoperative length of hospital stay (LOS) with SPPB. The validity of SPPB scores were assessed by ROC curves to analyze the sensitivity and specificity for identifying elderly patients with prolonged hospitalization.

Results

Although SPPB and three components of SPPB all were independent risk factors of prolonged hospitalization, only the SPPB summary score and the 4-meter gait speed components showed good discriminative capabilities. A SPPB summary score cutoff of 10 provided the best accuracy for identifying prolonged hospitalization. The net benefit of model 2 (Model 1 combined with SPPB cut-off score) was better than that of the model 1 that had threshold probabilities of $30 \%-100 \%$.

Conclusions

This study provided evidence for the clinical utility of the SPPB summary score and 4MGS test for prolonged hospitalization in elderly patients undergoing CABG. These tests also showed potential as a screening test for identifying elderly patients undergoing $C A B G$ who are at risk of prolonged hospitalization mobility.

\section{Introduction}

The elderly comprise the fastest growing segment of the global population. They are more susceptible to most diseases than younger adults and undergo surgery twice as often as their younger counterparts, and they are therefore the leading consumers of healthcare services. ${ }^{1}$ Older adults are commonly at high risk for worsening health and functional decline. Poor functional status has important consequences as it has been associated with the risk of longer hospital stay, home care placement, and mortality. ${ }^{2}$

CABG is the definitive surgical treatment for coronary artery disease and can be performed with a low incidence of morbidity and mortality. Despite improvements in medical care, post-operative medical complications are more frequent in the elderly population. ${ }^{3}$ There is always a tendency to search for additional and more reliable predictors. However, current risk prediction models, including the European System for Cardiac Operative Risk Evaluation (Euro SCORE), do not include objective measures for frailty. ${ }^{4}$ Frailty is increasingly recognized as a unique domain of health status that can be a marker of decreased reserves and resultant vulnerability in older patients. ${ }^{6}$ The use of frailty as a risk factor or determinant in predicting an adverse postsurgical outcome is evolving. In nonsurgical populations, this phenotype has been associated with adverse health outcomes. However, there have been few scientific studies that show the implications of frailty for CABG. ${ }^{4-10}$ We hypothesized that frailty predicts adverse postsurgical outcome in older CABG patients.

Impaired physical function is an important part and predictor of frailty. SPPB is one of the most commonly used instruments for measuring physical performance in population studies of aging. ${ }^{11}$ It has gained popularity in the past few years because compared with self-report measures, it is a standardized objective tool, it is rapid and simple to conduct, and it is less influenced by cultural and educational backgrounds. The SPPB represents the sum of the results from three component tests of functional relevance: standing balance, 4MGS, and 5STS. ${ }^{12}$ Research has shown that poor performance on SPPB is highly 
predictive of subsequent disability and mortality. ${ }^{13}$ SPPB has had limited use in hospitalized older patients. In recent studies, Volpato S. et al. ${ }^{2}$ and Clark K. et al. ${ }^{14}$ demonstrated the short-term predictive value of SPPB in older patients admitted to hospital.

Nevertheless, the predictive value of SPPB in elderly patients undergoing CABG has not been investigated. The primary aim of this paper was to determine the clinical utility of the SPPB summary score for prolonged postoperative LOS in patients undergoing CABG. A secondary aim was to assess whether any component of the SPPB might be equivalent to the full test battery in terms of clinical utility and value for postoperative LOS.

\section{Methods}

\section{Participants}

This prospective observational cohort study was conducted in patients aged $\geq 60$ who had isolated CABG at our institution between May 2017 and May 2018. Populations of our study were performed by the same surgical team in Tianjin, China. Exclusion criteria were a history of cerebrovascular accident; presence or history of neuromuscular disorders, other active severe diseases (e.g., cancer, kidney disease, and chronic systemic inflammatory diseases), cognitive deterioration, New York Heart Association (NYHA) functional class IV, or a limitation in walking. The performance-based measures of physical function and interview were assessed at hospital admission (within 48 hours after hospital admission, 2 hour after meal in the morning). And about 5 days later, they would then undergo CABG. During a 1-year period, a consecutive sample of eligible patients was identified from patient health examinations. Overall, 188 subjects from were identified. Patients who died in the operating room or surgical ward before discharge were excluded. For patients who underwent more than one CABG surgery, the final selection included only the most recent procedure. After surgery, the patients who stayed in the hospital > 8 days were classified as having a prolonged LOS. ${ }^{15}$ This study was approved by the Ethics Committee at Tianjin Medical University, China. Informed written consent was obtained directly from participants.

\section{Short Physical Performance Battery}

This study uses methodology from a previously published and the measure of SPPB is composed of three timed tests ${ }^{12}$ : a) standing balance tests, b) 4MGS test, and c) 5STS test. The standing balance tests require participants to maintain, for 10 seconds each, position with side-by-side position, semi-tandem position, and tandem position. The 4MGS measured the time needed to walk $4 \mathrm{~m}$ at a typical pace. This test was repeated twice and the analysis used the shorter time of the two. The 5STS required participants to stand up and sit down five times as quickly as possible, with their hands folded across their chest. Each of the three performance assessments was assigned categorical scores (range: $0-4$ ) and the summary ranging between 0 and 12 .

\section{Covariates}

Data were collected prospectively during the patients' admission by interviews and physical examinations on the following variables: age, sex, BMI, waist hip rate (WHR), educational level, occupation, current diagnoses, left ventricular ejection fraction (LVEF), NYHA classification, history of smoking and drinking, daily physical activity, psychological conditions, and history of hypertension, hyperlipidemia and diabetes were recorded before surgery. Information on smoking (never, former smoker, and current smoker) and drinking (never, former drinker, everyday drinker, and occasional drinker) was obtained from a questionnaire survey. Physical activity was assessed with the short form of the International Physical Activity Questionnaire (IPAQ), in Chinese. ${ }^{16}$ Psychological conditions were assessed with Generalized Anxiety Disorder scale (GAD-7) ${ }^{17}$ and Patient Health Questionnaire $(\mathrm{PHQ}-9)^{18}$. Surgery situations obtained from medical records included duration of surgery, number of bypass, bypass time, and length of stay in intensive care unit (ICU) and indwelling drainage tube. Postoperative complication 
was defined as the occurrence of any of the following events: atrial fibrillation, myocardial infarction, re-intubation, and renal failure, stroke, anemia, hemorrhage, infection, and sternal problems.

\section{Statistical analysis}

Demographic and clinical variables were presented as means \pm SD median, (interquartile range) or as percentages in table 1. Differences between these variables were examined by ANOVA (continuous variables) or chi-squared test (categorical variables), and differences were defined as significant when $P<0.05$. The simple logistic regression analysis was used to examine the independent influence of risk factors on postoperative LOS; the odds ratio (OR) and 95\% Confidence Interval (Cl) were computed. We performed multiple logistic regression analyses with a backward-stepwise procedure to test SPPB and three SPPB components as an independent predictor of postoperative LOS in table 2. Multiple logistic regression analyses of model 1 was adjusted for sex, age, BMI, LVEF, NYHA classification, number of bridge vessels, IPAQ, smoking, drinking, illiteracy, farming, depression, anxiety, diabetes, hypertension, hyperlipidemia, longer duration of surgery, bypass time, length of stay in ICU and postoperative complications. And the logistic regression analyses of model 2 combined model 1 with SPBB cores; model 3 combined model 1 with standing balance tests scores; model 4 combined model 1 with 4MGS scores; model 5 combined model 1 with 5STS scores.

In addition, the receiver operative characteristic (ROC) analysis allowed us to define the best cutoff score for discriminating prolonged hospitalization, based on the sensitivity (Se) and specificity (Sp) values associated with the scores of the various SPPB component tests. We assessed the sensitivity of the SPPB summary score and its three components to discriminate patients with prolonged hospitalization from those without, and present in the table 3 . Decision curve analysis was applied to calculate the net benefit of the screening model. All analyses were performed with the SPSS statistical software program (SPSS Inc., China) and R (R Foundation for Statistical Computing, v3.5.2). $P<0.05$ was considered statistically significant.

\section{Result}

The baseline characteristics, preoperative data, and outcome variables of the CABG patients according to postoperative LOS are presented in Table 1. Overall, 145/188 (77.2\%) patients were discharged from the hospital undergoing bypass surgery in $\leq$ 8 days, and $43(22.8 \%)$ patients spent > 8day in the hospital after CABG. In all the patients studied, the postoperative LOS ranged from 4 to 28 days (mean \pm SD, $7.62 \pm 3.72$ days) with a median of 7 days. Patients with a prolonged LOS (postoperative LOS day > 8days) were more likely have lower physical activity, longer operation time, bypass time and LOS in the ICU. Patients with prolonged postoperative LOS had a statistically significant lower SPPB score compared with patients with postoperative LOS $\leq 8$ days $(P<0.05)$.

After adjusting for potential confounders, SPPB and individual components of SPPB (standing balance test, the 4MGS test, and the 5STS test) were shown to be an independent risk factor for prolonged postoperative LOS with OR of 0.68 ( $95 \% \mathrm{Cl}$ of $0.56-0.83)$, OR of 0.37 ( $95 \% \mathrm{Cl}$ of $0.23-0.60)$, OR of 0.26 ( $95 \% \mathrm{Cl}$ of $0.14-0.48)$ and $0.54(95 \% \mathrm{Cl}$ of $0.37-0.79)$, respectively. The multivariable model 1 contained twenty variables (e.g. sex, age, BMI etc.) with a pseudo r-squared value of 0.267 . The three further regression models including the physical performance had pseudo r-squared values of: SPPB 0.426 (Model 2); standing balance test 0.368 (Model 3); 4MGS 0.395 (Model 4); 5STS 0.347 (Model 5).

For the SPPB summary score, a cut-off score of $9.5(<10)$ provided the most accurate discrimination of prolonged postoperative LOS. With this cut-off score, we calculated Se and Sp values of 0.847 and 0.647 , respectively (table 3 ). Only 4MGS showed good discrimination (AUC: 0.703 ). The ROC curve plotted using sensitivity and specificity calculated from SPPB and 4MGS with hospitalization stay after CABG and was shown as Figure 1. The net benefit of model 2 was better than that of the model 1 that had threshold probabilities of 30\%-100\% (Figue 2 Model 1: clinical parameter (e.g. sex, age, BMI etc.); Model 2: Model 1 combined with SPPB cut-off score)

\section{Discussion}


Our findings provided evidence for the clinical utility of the full performance test battery and 4MGS test as clinical tools for assessing prolonged postoperative LOS in patients with CABG. Though the AUC values of standing balance and 5STS were $<0.7$, these two components were also independent risk factors for postoperative hospitalization. To our knowledge, this was the first study to assess whether one of the three components of the SPPB might provide value equivalent to the SPPB for assessing patients with CABG.

\section{SPPB scores predictive of hospital length of stay}

Despite a large body of evidence demonstrating the predictive value of different physical performance tests in terms of various adverse outcomes in elders, ${ }^{13}$ the use of physical performance measures in the cardiac surgery clinical setting has received little attention thus far. ${ }^{46791019}$ This study shows that, SPPB score evaluated at hospital admission provided the most accurate discrimination of prolonged postoperative LOS. Our results confirm and extend, at a clinical level, the findings of previous studies. In particular, our findings are consistent with the study by Clark K. and colleagues, who demonstrated that SPPB is a valid indicator of functional and clinical status, and that the SPPB score at hospital admission is an independent predictor of the length of hospital stay. ${ }^{214}$ Physical performance is likely to capture the integrated and multisystemic effect on the health status of older persons, such as aging, comorbidity, disease severity, ${ }^{20-22}$ and so on. Like other biomarkers, SPPB might be considered to be a nonspecific but highly sensitive indicator of global health status reflecting several underlying physiological impairments. ${ }^{23}$ Some of these patients with lower SPPB scores are likely to be more frail, vulnerable, and clinically unstable and therefore at greater risk of prolonged postoperative LOS. Also, patients undergoing CABG those presented with worse SPPB scores might be at increased risk of prolonged postoperative LOS due to the higher cardiopulmonary bypass and period of anoxia during surgery. This hypothesis would explain why SPPB had better prognostic value compared to other individual indicators.

In addition, we found that the SPPB summary score, a cutoff score of $<10$, provided the most accurate determination of the prolonged postoperative LOS. Moreover, this cutoff value may also be used to identify older persons with chronic obstructive pulmonary disease at high risk of functional impairment ${ }^{24}$ and mobility limitations ${ }^{25}$. Also, SPPB scores $<7$ are generally associated with a higher fall rate in the elderly; an SPPB score $<5$ could identify patients at major risk of death after discharge from acute care hospitals. ${ }^{26}$ Thus, different cutoff values of the SPPB score are needed for different kinds of diseases. This is the first study to explore the cutoff value of SPPB in CABG patients.

The potential applications of the findings from the present study include their clinical value. For example, the SPPB scoring system was feasible to perform in a busy surgical practice, taking ten minutes to conduct the assessment. Once a patient has been identified as impaired physical function, surgeons can integrate impaired physical function into their discussions of the risks and benefits of surgery. In addition, patients can benefit from interventions to reduce risk, such as a preoperative increase in balance, walking ability, and strength training. In conclusion, well-designed clinical studies will be needed to develop targeted risk reduction strategies for patients with impaired physical function.

\section{Three components of SPPB scores predictive of hospital LOS}

In the present study, we tested whether any of the three SPPB components (standing balance, 4MGS, and 5STS) were suitable for identifying patients at risk of prolonged postoperative LOS. We found that the 4MGS test had the largest AUC value in an ROC analysis than the other two components of SPPB scores. A study by Afilalo $\mathrm{J}$ et al. ${ }^{27}$ showed gait speed is an incremental predictor of mortality and major morbidity in elderly patients undergoing cardiac surgery. Besides that, a new study demonstrated a significant association between preoperative gait speed and increased length of hospital stay following cardiac surgery, which was consistent with the results of our own papers. ${ }^{14}$ Gait speed is an easy-to-determine, valid, and reliable assessment that has been frequently cited as a useful clinical indicator of an individual's physical frailty and future morbidity and mortality. ${ }^{28}$ in addition to the 30 -day mortality following transcatheter aortic valve replacement, ${ }^{29}$ with good 
inter-rater and retest reliability. From this point of view, our findings reinforce the concept that gait speed has long been an established marker for exercise capacity in elderly patients and may prove useful as part of a multidimensional assessment of physical performance in CABG patients. Thus, assessing physical performance using a standardized definition can help patients and physicians make more informed decisions.

Though the AUC values of standing balance and 5STS were $<0.7$, this study showed that these two components were also independent risk factors for LOS in patients with CABG and the relationship remained significant after adjustment for confounding factors. Impaired balance was well-recognized as being common in elderly people with impaired function. Since strength, muscle mass and balance are all closely interlinked; 5STS is an appropriate indicator of lower limb muscle strength and balance. Many cross-sectional and prospective studies have revealed that muscle strength is an indicator of functional decline in older people. ${ }^{30}$ Skeletal muscle is the largest tissue in the body, acting as an important contributor to whole body glucose and protein metabolism, and has a role to maintain nutritional homeostasis and provides nutrients for the immune system and the rapid repair of tissue. ${ }^{31}$ Poor nutritional status increased risk for adverse clinical outcome, ${ }^{32}$ which could lead to an increased risk of prolonged postoperative LOS. Thus, strength and balance are closely related and are important for improving dynamic balance and increasing strength, which both help to prevent physical functions decrease in older patients who undergo elective CABG.

Despite our findings that the SPPB generally shows good clinical utility, several limitations should be noted. First, this study was restricted to a single region of China; therefore, the discriminative ability of the SPPB may not be generalizable to different settings. Second, owing to the study on the prediction of SPPB in CABG patients is a relative new research and limited study spanning time, and lack of substantial previous article. Temporal, geographical and external validation cannot build at present stage. To overcome this problem, sustaining follow up study in our hospital and multi-center research are conducting, hoping to enhance validation. Besides, external validation in large cohorts is also needed before SPBB into practice to make clinical decision. Third, though we adopted all elderly who had isolated CABG at our institution between May 2017 and May 2018 and eliminated all who meet any exclusion criteria, the elderly population with an average age of $66.5 \pm 4.8$ in our study was relatively healthy and not representative of a real life elderly cardiac patient population undergoing cardiac surgery. So our results may not be replicated in sicker elderly populations, where SPPB may lose predictive power for outcome compared to other instruments of the multidimensional geriatric evaluation. Therefore the proposed SPPB cut off point of 10 for prediction of longer hospitalization may not be applicable in sicker and senile patients undergoing CABG. At last, we only explored the short-term predictive value of SPPB. Factors indicative of more long-term clinical value need to be assessed in follow-up studies.

\section{Conclusion}

This study provided evidence for the clinical utility of the SPPB summary score and the 4MGS test for assessing LOS in elderly patients undergoing CABG. Our results showed that these tests had equivalent potential as screening tools for identifying patients at risk of prolonged postoperative LOS. Further evidence of the predictive utility of SPPB may lead to SPPB analysis as a standard prescreening tool for CABG, which may in turn assist physical therapists and other clinicians in identifying those patients who may need more intensive services, preplanning for a longer duration of hospital stay, or have a need for placement in a skilled nursing facility before surgery.

\section{Declarations}

\section{Ethical approval and consent participate}

This study was approved by the Tianjin Medical University ethics committee (TJMU18052401). Every participant was fully informed of research nature. All participants signed an informed consent form to participate before data collection.

\section{Consent for publication}

Page $7 / 13$ 
Not applicable

Availability of data and materials

The datasets used and/or analysed during the current study are de-identified and available from the corresponding author on reasonable request.

Competing interests

The authors declare that they have no competing interests.

Funding

This study was supported by Tianjin Municipal Science and Technology Commission grant number [16ZXMJSY00070], and Health and Family Planning Commission of Binhai New Area grant number [2017BWKZ005].

\section{Authors' contributions}

All authors contributed to the study conception and design. Material preparation, data collection and analysis were performed by QG, XC L, ZL, YL, JJ L, LF, XY, XC, HY, LH, LW, JL, XL, BS, BY, ML, HC, LT, MG, ZW and TC. The first draft of the manuscript was written by $\mathrm{PH}$ and all authors commented on previous versions of the manuscript. All authors read and approved the final manuscript.

Acknowledgements

The authors thank all the staff who had provided helping to our study in TEDA international cardiovascular hospital and Tianjin Medical University.

\section{References}

1. Kelley AS, McGarry K, Gorges R, et al. The burden of health care costs for patients with dementia in the last 5 years of life. Ann Intern Med 2015;163(10):729-36.

2. Volpato $S$, Cavalieri $M$, Guerra $G$, et al. Performance-based functional assessment in older hospitalized patients: feasibility and clinical correlates. J Gerontol A Biol Sci Med Sci 2008;63(12):1393-8.

3. Polanczyk CA, Marcantonio E, Goldman L, et al. Impact of age on perioperative complications and length of stay in patients undergoing noncardiac surgery. Ann Intern Med 2001;134(8):637-43.

4. Ad N, Holmes SD, Halpin L, et al. The Effects of Frailty in Patients Undergoing Elective Cardiac Surgery. J Card Surg 2016;31(4):187-94.

5. Afilalo J, Mottillo S, Eisenberg MJ, et al. Addition of frailty and disability to cardiac surgery risk scores identifies elderly patients at high risk of mortality or major morbidity. Circ Cardiovasc Qual Outcomes 2012;5(2):222-8.

6. Makary MA, Segev DL, Pronovost PJ, et al. Frailty as a predictor of surgical outcomes in older patients. J Am Coll Surg 2010;210(6):901-8.

7. Wilson CM, Kostsuca SR, Boura JA. Utilization of a 5-Meter Walk Test in Evaluating Self-selected Gait Speed during Preoperative Screening of Patients Scheduled for Cardiac Surgery. Cardiopulm Phys Ther J 2013;24(3):36-43.

8. Herman CR, Buth KJ, Legare JF, et al. Development of a predictive model for major adverse cardiac events in a coronary artery bypass and valve population. J Cardiothorac Surg 2013;8:177.

9. Cervera R, Bakaeen FG, Cornwell LD, et al. Impact of functional status on survival after coronary artery bypass grafting in a veteran population. Ann Thorac Surg 2012;93(6):1950-4; discussion 54-5. 
10. Sundermann S, Dademasch A, Praetorius J, et al. Comprehensive assessment of frailty for elderly high-risk patients undergoing cardiac surgery. Eur J Cardiothorac Surg 2011;39(1):33-7.

11. Guralnik JM, Ferrucci L, Simonsick EM, et al. Lower-extremity function in persons over the age of 70 years as a predictor of subsequent disability. N Engl J Med 1995;332(9):556-61.

12. Bernabeu-Mora R, Medina-Mirapeix F, Llamazares-Herran E, Garcia-Guillamon G, Gimenez-Gimenez LM, Sanchez-Nieto JM: The Short Physical Performance Battery is a discriminative tool for identifying patients with COPD at risk of disability. Int J Chron Obstruct Pulmon Dis 2015, 10:2619-2626.

13. Gomez JF, Curcio CL, Alvarado B, et al. Validity and reliability of the Short Physical Performance Battery (SPPB): a pilot study on mobility in the Colombian Andes. Colomb Med (Cali) 2013;44(3):165-71.

14. Clark K, Leathers T, Rotich D, et al. Gait Speed Is Not Associated with Vasogenic Shock or Cardiogenic Shock following Cardiac Surgery, but Is Associated with Increased Hospital Length of Stay. Crit Care Res Pract 2018;2018:1538587.

15. Hagg S, Alserius T, Noori P, et al. Blood levels of dual-specificity phosphatase- 1 independently predict risk for postoperative morbidities causing prolonged hospitalization after coronary artery bypass grafting. Int J Mol Med 2011;27(6):851-7.

16. Han P, Kang L, Guo Q, et al. Prevalence and Factors Associated With Sarcopenia in Suburb-dwelling Older Chinese Using the Asian Working Group for Sarcopenia Definition. J Gerontol A Biol Sci Med Sci 2016;71(4):529-35.

17. Herr NR, Williams JW, Jr., Benjamin S, et al. Does this patient have generalized anxiety or panic disorder?: The Rational Clinical Examination systematic review. JAMA 2014;312(1):78-84.

18. Kawada T. Depression screening by Patient Health Questionnaire in patients with cardiac surgery. Int J Cardiol 2016;212:355-6.

19. Afilalo J, Lauck S, Kim DH, et al. Frailty in Older Adults Undergoing Aortic Valve Replacement: The FRAILTY-AVR Study. $J$ Am Coll Cardiol 2017;70(6):689-700.

20. Karlamangla A, Tinetti M, Guralnik J, et al. Comorbidity in older adults: nosology of impairment, diseases, and conditions. J Gerontol A Biol Sci Med Sci 2007;62(3):296-300.

21. Atkinson HH, Rosano C, Simonsick EM, et al. Cognitive function, gait speed decline, and comorbidities: the health, aging and body composition study. J Gerontol A Biol Sci Med Sci 2007;62(8):844-50.

22. Forrest KY, Zmuda JM, Cauley JA. Correlates of decline in lower extremity performance in older women: A 10-year followup study. J Gerontol A Biol Sci Med Sci 2006;61(11):1194-200.

23. Morley JE. Mobility performance: a high-tech test for geriatricians. J Gerontol A Biol Sci Med Sci 2003;58(8):712-4.

24. Patel MS, Mohan D, Andersson YM, et al. Phenotypic characteristics associated with reduced short physical performance battery score in COPD. Chest 2014;145(5):1016-24.

25. Bernabeu MR, Medina,M.F., Llamazares,H.E., García,G.G., Giménez-Giménez LM, Sánchez,Nieto JM. The Short Physical Performance Battery is a discriminative tool for identifying patients with COPD at risk of disability Int J Chron Obstruct Pulmon Dis 2016;11:623.

26. Corsonello A, Lattanzio F, Pedone C, et al. Prognostic significance of the short physical performance battery in older patients discharged from acute care hospitals. Rejuvenation Res 2012;15(1):41-8.

27. Afilalo J, Eisenberg MJ, Morin JF, et al. Gait speed as an incremental predictor of mortality and major morbidity in elderly patients undergoing cardiac surgery. J Am Coll Cardiol 2010;56(20):1668-76.

28. Munoz-Mendoza CL, Cabanero-Martinez MJ, Millan-Calenti JC, et al. Reliability of 4-m and 6-m walking speed tests in elderly people with cognitive impairment. Arch Gerontol Geriat 2011;52(2):E67-E70.

29. Alfredsson J, Stebbins A, Brennan JM, et al. Gait Speed Predicts 30-Day Mortality After Transcatheter Aortic Valve Replacement: Results From the Society of Thoracic Surgeons/American College of Cardiology Transcatheter Valve Therapy Registry. Circulation 2016;133(14):1351-9.

30. Gariballa S, Alessa A. Impact of poor muscle strength on clinical and service outcomes of older people during both acute illness and after recovery. BMC Geriatr 2017;17(1):123.

Page 9/13 
31. Lightfoot A, McArdle A, Griffiths RD. Muscle in defense. Crit Care Med 2009;37(10 Suppl):S384-90.

32. Sorensen J, Kondrup J, Prokopowicz J, et al. EuroOOPS: an international, multicentre study to implement nutritional risk screening and evaluate clinical outcome. Clinical nutrition (Edinburgh, Scotland) 2008;27(3):340-9.

\section{Tables}

Table1. Characteristics of study participants according to postoperative stay

\begin{tabular}{|c|c|c|c|}
\hline Characteristic & $\begin{array}{c}\text { Normal ( } \leq 8 \text { days }) \\
\mathbf{N}=145\end{array}$ & $\begin{array}{l}\text { Prolonged(>8 days) } \\
\qquad \mathrm{N}=43\end{array}$ & $P$ value \\
\hline \multicolumn{4}{|l|}{ Genaral data } \\
\hline Age (y) & $66.24 \pm 4.47$ & $67.56 \pm 5.97$ & 0.151 \\
\hline $\operatorname{Sex}(\%)$ & & & 0.373 \\
\hline Female & 33.1 & 41.2 & \\
\hline Male & 66.9 & 58.8 & \\
\hline BMI $\left(\mathrm{kg} / \mathrm{m}^{2}\right)$ & $26.03 \pm 2.96$ & $25.70 \pm 2.81$ & 0.565 \\
\hline WHR & $0.97 \pm 0.08$ & $0.97 \pm 0.06$ & 0.944 \\
\hline LVEF (\%) & $58.17 \pm 6.12$ & $57.79 \pm 4.32$ & 0.735 \\
\hline NYHA classification system (\%) & & & 0.553 \\
\hline Class I & 80.6 & 88.2 & \\
\hline Class II & 16.0 & 8.8 & \\
\hline Class III & 3.5 & 2.9 & \\
\hline Number of bridge vessels (n) & $3.6 \pm 0.9$ & $3.7 \pm 0.8$ & 0.673 \\
\hline IPAQ(MET) & $2820(1386-6746 \square$ & 2773(1318-6426) & 0.817 \\
\hline Smoking (\%) & 39.0 & 35.3 & 0.923 \\
\hline Drinking (\%) & 23.3 & 18.8 & 0.529 \\
\hline Illiteracy (\%) & 16.5 & 22.6 & 0.427 \\
\hline Farming $(\%$ & 39.8 & 39.4 & 0.962 \\
\hline Depression (\%) & 10.9 & 9.1 & 0.765 \\
\hline Anxiety (\%) & 10.2 & 6.1 & 0.463 \\
\hline Diabetes (\%) & 37.8 & 38.3 & 0.959 \\
\hline Hypertension (\%) & 69.1 & 82.4 & 0.142 \\
\hline Hyperlipidemia (\%) & 3.1 & 4.7 & 0.699 \\
\hline Physical Performance & 5.3 & 12.0 & 0.250 \\
\hline SPPB (score) & $10.69 \pm 1.57$ & $8.65 \pm 2.73$ & $<0.001$ \\
\hline Standing balance test (score) & $3.72 \pm 0.59$ & $3.09 \pm 1.21$ & $<0.001$ \\
\hline 4MGS (score) & $3.79 \pm 0.49$ & $3.15 \pm 0.96$ & $<0.001$ \\
\hline 5STS (score) & $3.17 \pm 0.96$ & $2.41 \pm 1.19$ & $<0.001$ \\
\hline \multicolumn{4}{|l|}{ Operative data } \\
\hline Duration of operation (h) & $3.98 \pm 0.88$ & $4.61 \pm 1.43$ & 0.002 \\
\hline Bypass time (min) & $99 \pm 37$ & $109 \pm 47$ & 0.324 \\
\hline Length of stay in ICU (h) & $42.80(39.49-44.41 \square$ & 43.83(42.20-62.84ם & 0.007 \\
\hline $\begin{array}{l}\text { Length of indwelling drainage tube } \\
\text { (h) }\end{array}$ & 64.35(50.51-67.56ロ & $65.96(43.92-127.05 \square$ & 0.169 \\
\hline Postoperative complication (\%) & 8.3 & 26.5 & 0.003 \\
\hline
\end{tabular}

BMI =body mass index; WHR =waist hip rate; LVEF =left ventricular ejection fraction; NYHA = New York Heart Association; IPAQ = international physical activity questionnaire; MET/wk =metabolic equivalent task minutes per week; SPPB =The Short Physical Performance Battery; 4MGS =4-meter gait speed; 5STS =five-repetition sit-to-stand motion; ICU= intensive care unit. 
Table 2 Results of Cox proportional hazards analysis for postoperative stay

\begin{tabular}{|c|c|c|c|c|c|c|c|c|c|c|c|c|}
\hline & \multicolumn{2}{|c|}{ Univariate analysis } & \multicolumn{10}{|c|}{ Multivariate analysis } \\
\hline & OR & $P$ & Model 1 & & Model 2 & & Model & 3 & Model 4 & & Model 5 & \\
\hline & $\begin{array}{l}(95 \% \\
\text { CI) }\end{array}$ & & $\begin{array}{l}\text { OR } \\
(95 \%\end{array}$ & & $\begin{array}{l}\text { OR } \\
(95 \%\end{array}$ & & $\begin{array}{l}\text { OR } \\
(95 \%\end{array}$ & & $\begin{array}{l}\text { OR } \\
(95 \%\end{array}$ & & $\begin{array}{l}\text { OR } \\
(95 \%\end{array}$ & \\
\hline & & & CI) & $P$ & CI) & $P$ & CI) & $P$ & CI) & $P$ & CI) & $P$ \\
\hline & $\begin{array}{l}1.05 \\
(0.98- \\
1.14)\end{array}$ & 0.146 & & & & & & & & & & \\
\hline & $\begin{array}{l}1.45 \\
(0.67- \\
3.11)\end{array}$ & 0.347 & & & & & & & & & & \\
\hline 12) & $\begin{array}{l}0.96 \\
(0.84- \\
1.10)\end{array}$ & 0.559 & & & & & & & & & & \\
\hline & $\begin{array}{l}0.97 \\
(0.92- \\
1.03)\end{array}$ & 0.269 & & & & & & & & & & \\
\hline tion & $\begin{array}{l}0.67 \\
(0.27- \\
1.64)\end{array}$ & 0.380 & & & & & & & $\begin{array}{l}0.43 \\
(0.15- \\
1.26)\end{array}$ & 0.125 & & \\
\hline T) & $\begin{array}{l}0.98 \\
(0.97- \\
1.02)\end{array}$ & 0.973 & & & & & & & & & & \\
\hline$(\%)$ & $\begin{array}{l}1.08 \\
(0.70- \\
1.68)\end{array}$ & 0.731 & & & & & & & & & & \\
\hline$(\%)$ & $\begin{array}{l}1.22 \\
(0.83- \\
1.81)\end{array}$ & 0.312 & & & & & & & & & & \\
\hline$(\%)$ & $\begin{array}{l}1.43 \\
(0.56- \\
3.71)\end{array}$ & 0.453 & & & & & & & & & & \\
\hline$\% \square$ & $\begin{array}{l}1.06 \\
(0.49- \\
2.30)\end{array}$ & 0.877 & & & & & & & & & & \\
\hline in (\%) & $\begin{array}{l}0.83 \\
(0.23- \\
3.05)\end{array}$ & 0.782 & & & & & & & & & & \\
\hline$\%)$ & $\begin{array}{l}0.58 \\
(0.13- \\
2.69)\end{array}$ & 0.486 & & & & & & & & & & \\
\hline$(\%)$ & $\begin{array}{l}1.06 \\
(0.49- \\
2.30)\end{array}$ & 0.877 & & & & & & & & & & \\
\hline sion & $\begin{array}{l}2.19 \\
(0.85- \\
5.66)\end{array}$ & 0.105 & & & & & & & & & & \\
\hline demia & $\begin{array}{l}1.44 \\
(0.17- \\
12.33)\end{array}$ & 0.742 & & & & & & & & & & \\
\hline of & $\begin{array}{l}1.09 \\
(0.70- \\
1.71)\end{array}$ & 0.693 & & & & & & & & & & \\
\hline $\begin{array}{l}\text { of } \\
(\mathrm{h})\end{array}$ & $\begin{array}{l}1.71 \\
(1.20- \\
2.43)\end{array}$ & 0.003 & $\begin{array}{l}1.54 \\
(1.02- \\
2.34)\end{array}$ & 0.040 & $\begin{array}{l}1.55 \\
(0.99- \\
1.04)\end{array}$ & 0.054 & $\begin{array}{l}1.59 \\
(1.02- \\
2.47)\end{array}$ & 0.041 & $\begin{array}{l}1.65 \\
(1.03- \\
2.63)\end{array}$ & 0.041 & $\begin{array}{l}1.52 \\
(0.99- \\
2.32)\end{array}$ & 0.054 \\
\hline time & $\begin{array}{l}1.01 \\
(0.99- \\
1.02)\end{array}$ & 0.321 & & & & & & & & & & \\
\hline e stay & $\begin{array}{l}1.03 \\
(1.01- \\
1.05)\end{array}$ & 0.008 & $\begin{array}{l}1.02 \\
(1.01- \\
1.04)\end{array}$ & 0.046 & $\begin{array}{l}1.01 \\
(0.99- \\
1.04)\end{array}$ & 0.104 & $\begin{array}{l}1.02 \\
(1.01- \\
1.05)\end{array}$ & 0.035 & $\begin{array}{l}1.02 \\
(0.99- \\
1.05)\end{array}$ & 0.099 & $\begin{array}{l}1.02 \\
(0.99- \\
1.04)\end{array}$ & 0.128 \\
\hline tion & $\begin{array}{l}3.96 \\
(1.51- \\
10.39)\end{array}$ & 0.005 & $\begin{array}{l}2.71 \\
(0.93- \\
7.92)\end{array}$ & 0.066 & $\begin{array}{l}3.09 \\
(0.85- \\
10.07)\end{array}$ & 0.061 & $\begin{array}{l}3.28 \\
(1.07- \\
10.65)\end{array}$ & 0.037 & $\begin{array}{l}3.56 \\
(1.06- \\
11.96)\end{array}$ & 0.041 & $\begin{array}{l}3.11 \\
(1.01- \\
9.67)\end{array}$ & 0.049 \\
\hline re) & $\begin{array}{l}0.63 \\
(0.51- \\
0.77)\end{array}$ & $<0.001$ & -- & & $\begin{array}{l}0.68 \\
(0.56- \\
0.83)\end{array}$ & $<0.001$ & -- & & -- & & -- & \\
\hline st & $\begin{array}{l}0.44 \\
(0.28- \\
0.68)\end{array}$ & $<0.001$ & -— & & - - & & $\begin{array}{l}0.37 \\
(0.23- \\
0.60)\end{array}$ & $<0.001$ & - & & - - & \\
\hline re) & $\begin{array}{l}0.30 \\
(0.17- \\
0.51)\end{array}$ & $<0.001$ & - - & & -- & & - - & & $\begin{array}{l}0.26 \\
(0.14- \\
0.48)\end{array}$ & $<0.001$ & - - & \\
\hline re) & $\begin{array}{l}0.52 \\
(0.37- \\
0.74)\end{array}$ & $<0.001$ & - - & & - - & & - & & - - & & $\begin{array}{l}0.54 \\
(0.37- \\
0.79)\end{array}$ & 0.002 \\
\hline
\end{tabular}


Table 3 Cutoff point and sensibility of SPPB and individual components of SPPB according to ROC curve.

\begin{tabular}{lccc}
\hline Variable & Cutoff point & Sensibility & Specificity \\
\cline { 1 - 4 } SPPB & 9.5 & 0.847 & 0.647 \\
Standing balance test & 3.5 & 0.799 & 0.471 \\
4MGS & 3.5 & 0.833 & 0.559 \\
\hline 5STS & 2.5 & 0.771 & 0.559 \\
\hline
\end{tabular}

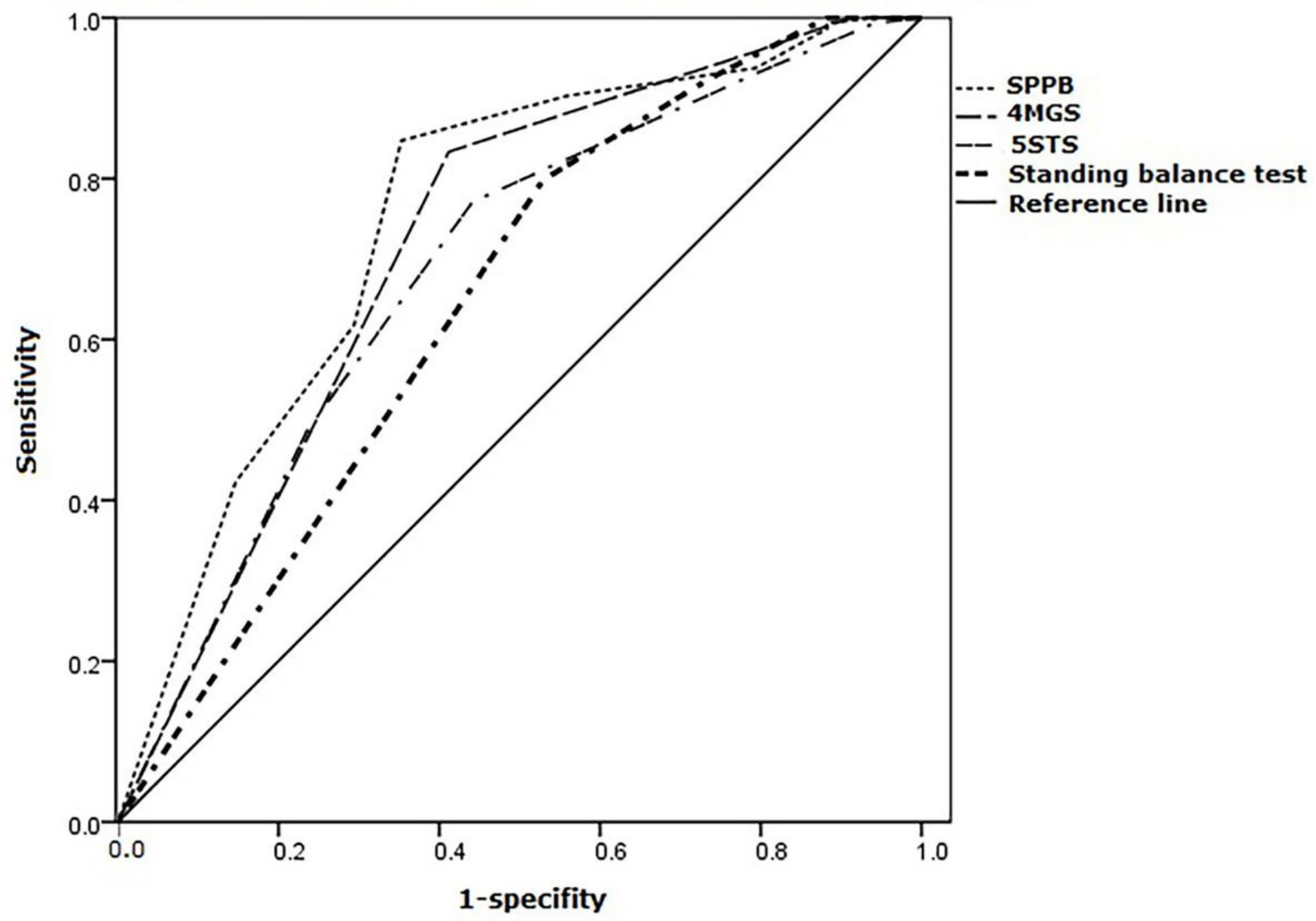

Figure 1

ROC curve plotted using sensitivity and specificity calculated from SPPB and its three components with hospitalization stay after CABG 


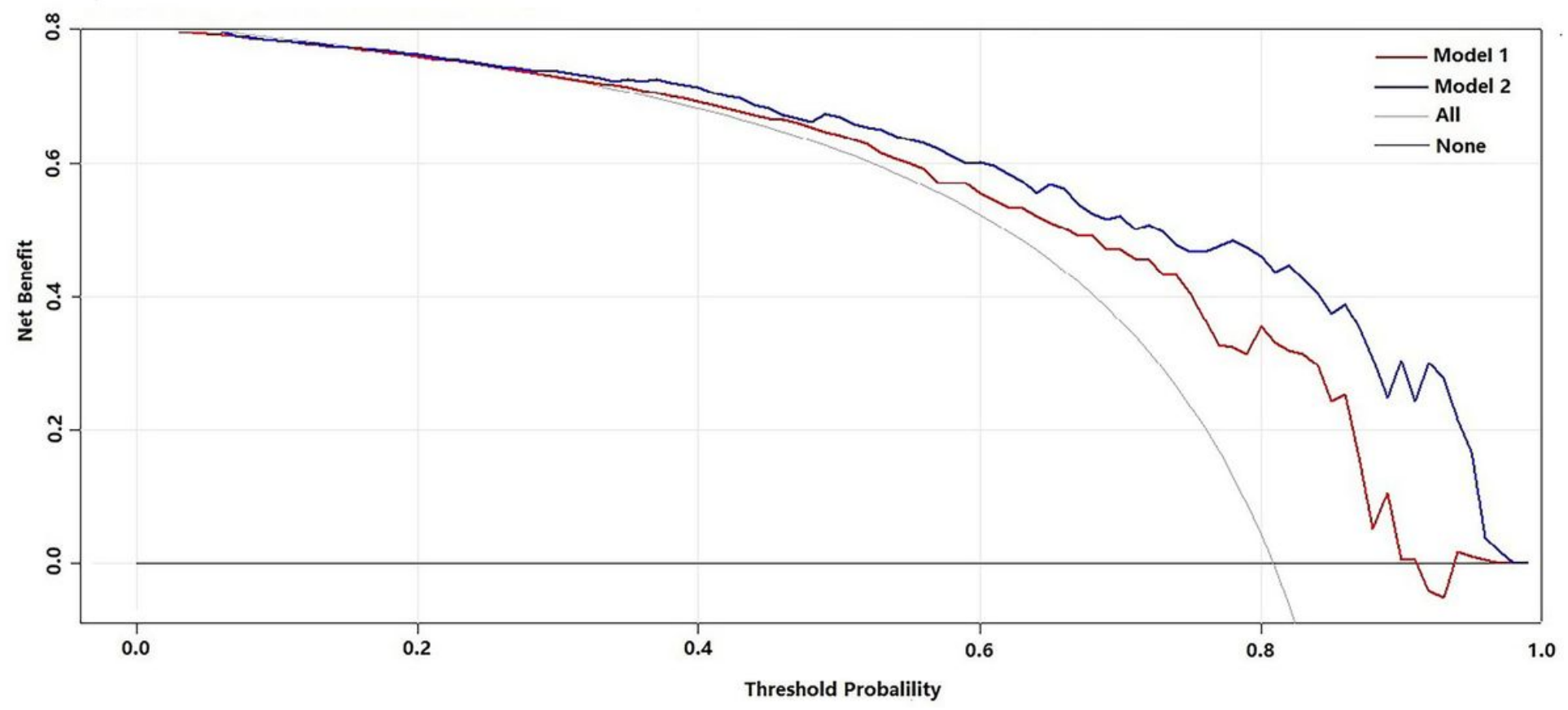

Figure 2

Decision curve analysis for the SPPB scores model (Model 1: clinical parameter (e.g. sex, age, BMI etc.); Model 2: Model 1 combined with SPPB cut-off score) 